\title{
Cholce between magnitudes of reward as a function of free vs forced trial procedures'
}

\author{
MARGARET F. BURRILL ${ }^{2}$ and NORMAN E. SPEAR, \\ Rutgers University, New Brunswick, N. J. 08903
}

Naive rats were trained on a $T$-maze spatial discrimination with four-pellets reward in the alternative designated "correct" and two pellets in the opposite alternative. For half the Ss, all trials were free while for the remainder, experience with the two alternatives was equated by means of forced trials. The group given forced trials acquired the discrimination significantly more rapidly than that given all free trials. Further, a significant positive correlation was observed between number of early choice alternations and later probability of a correct choice in the free-trial group.

Forced-trial procedures have frequently been employed in the study of discrimination learning to equate or systematically vary experience with alternatives so that the effects of variables such as magnitude, delay, or percentage of reinforcement might be studied without confounding the variables in question with differential experience. However, little consideration has been given to the question of what effect forced trials per se may have on animal discrimination learning.

Typical forcing procedures not only equate experience with alternatives but also ensure more runs to the less favorable alternative (LFA) than would occur with free trials. A number of studies (e.g., Lachman, 1961) have reported better discrimination performance by groups given larger proportions of runs to the LFA. It is quite reasonable to expect that as discrimination develops, runs to the LFA become increasingly frustrating and serve to build up avoidance of that alternative, thereby increasing the probability of choosing the more favorable alternative (MFA) on free trials. It might thus be predicted that forced-trial procedures, by increasing experience with, and thus avoidance of the LFA, might facilitate discrimination performance. The present experiment was designed to test this prediction.

\section{SUBJECTS}

Subjects were 20 naive female rats purchased from Sprague-Dawley in Madison, Wisconsin. The rats were about 60 days old at the start of training.

\section{APPARATUS}

The apparatus was a $\mathrm{T}$ maze painted flat black and with clear Plexiglas lids. It was 4 in. high and 4 in. wide at all points and consisted of a 16-in. stem and 40-in. arms, the last 18 in. of which constituted the goal boxes. Guillotine doors just beyond the choice point were used to prevent retracing and for forcing purposes.

\section{PREHANDLING}

Upon arrival from the supplier, the rats were housed in individual cages and placed on a daily food ration of $12 \mathrm{~g}$ ground Purina chow. Water was available in the home cages at all times. Beginning the day after arrival, Ss were given seven daily prehandling sessions which consisted of weighing and placement in a painted wooden box for a period of $4 \mathrm{~min}$. The box was partitioned into two compartments and Ss were handled in pairs, being picked up, stroked, and replaced in the box at least four times during each session. Food cups, later to be used in the maze, and containing four $45-\mathrm{mg}$ Noyes pellets were placed in the prehandling box. Any uneaten pellets plus one additional pellet were returned with $S$ to the home cage following handling.

\section{PROCEDURE}

Following prehandling, all Ss were given 150 training trials at six per day, with four Noyes $45-\mathrm{mg}$ pellets in the MFA (right or left randomly determined for each $S$ ) and two pellets in the LFA. For half the Ss, all trials were free, while for the remaining Ss, a schedule of forced and free trials was employed to equate experience with the two alternatives. For this group, the first and fifth trials each day were free, the second and sixth were forced opposite to the side chosen on the previous trial, and the third and fourth trials were forced one to the LFA and one to the MFA in predetermined random order.

\section{RESULTS AND DISCUSSION}

The probability of choosing the MFA on free trials or the corresponding Trials 1 and 5 for free-trial Ss has been plotted as a function of Blocks of Days in Fig. 1. Analysis of total errors on Trials 1 and 5 throughout the 25 days of training, revealed significantly more errors for the free- than for the forced-trial group $[t(18)=3.56, p<.01]$. A comparison was also made of days to the start of criterion run where criterion run was defined as 10 consecutive MFA choices on Trials 1 and 5. Two Ss in the free-trial group failed to meet this criterion and were assigned the maximum score of 25 days. Again, the free-trial group was found to be significantly inferior (more days to criterion) to the forced-trial group $[\mathrm{t}(18)=2.24, \mathrm{p}<.05]$.

It appears that the procedure of forcing trials to equate experience of alternatives does indeed facilitate discrimination between rewards at least under the conditions of the present experiment. This facilitation may be due in part to the greater number of LFA runs experienced by $S s$ in the forced-trial group. However, another possible interpretation of this finding was suggested by a post hoc analysis of the protocols of Ss given all free trials. It was noted that some Ss alternated their choices back and forth between the LFA and MFA much more frequently on early trials than other Ss. Furthermore, the same Ss who showed the greater tendency to alternate were those who acquired the discrimination most rapidly. To test the

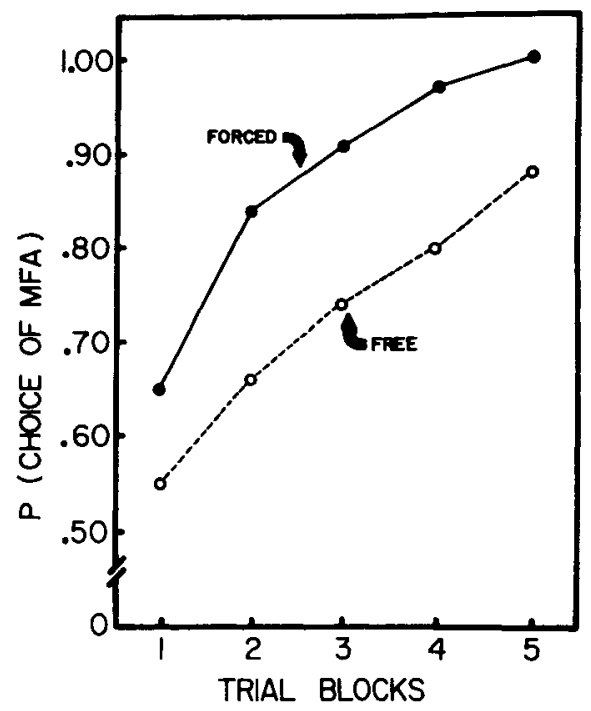

Fig. 1. Probability of choosing the more favorable alternative on Trials 1 and 5 each day during successive blocks of five days. 
reliability of this trend, correlation between number of early alternations (Trials 1-30) and total correct choices throughout training was computed. A significant positive correlation was found $[r(8)=+.68, p<.05]$. Of course it is conceivable, though unlikely, that strong alternation tendency may be an incidental characteristic of fast learners without contributing causally to rapid learning.

A positive relationship between alternation and discrimination performance also has been reported by Lester (1967). In Lester's study, rats who alternated more on a pretest of exploratory tendency learned a later $\mathrm{T}$ maze discrimination more rapidly than rats who alternated less on the pretest. Lester did not report whether the same difference in alternation obtained early in $T$ maze training, but it seems probable that it did. However, Clayton (1966) found no relationship between extent of alternation behayior (related to intertrial interval) and $T$ maze acquisition. It is not at all obvious why different results were obtained in Clayton's and the present experiment, but one possibility immediately suggests itself. This is the difference in problem difficulty in the two experiments. In Clayton's study, choice was between reward vs nonreward, and all groups acquired the discrimination within 36 trials. The present study involved reward for both alternatives, and learning was much slower. Perhaps this difference provides a clue to the mechanism underlying the observed relation between alternation and rate of acquisition in the free-trial group of the present study.

Why should frequent alternation facilitate a difficult discrimination? One possibility is that frequent alternation is accompanied by an increase in LFA experiences. But examination of the data obtained from free-trial animals revealed no correlation between number of early alternations and total errors on all trials for the same period. Thus, it appeared that alternation exerted a positive effect on acquisition performance not attributable to increased experi- ence with the LFA. Perhaps frequent alternation of stimuli, by providing many opportunities to compare stimuli in close temporal proximity, somehow served to draw attention to, and thus emphasize, the difference in reward magnitude associated with the LFA and MFA. Any advantage resulting thereby might reasonably be expected to exert greater influence in a difficult discrimination task than in a relatively simpler one, which would account for the discrepant results of this and the Clayton study.

Finally, might not the same factor be operating in the forced-trial condition? Frequent alternation is inherent in most forcing schedules. For example, the schedule employed in the present study, which is typical, involved a minimum of three alternations in each daily block of six trials. Perhaps the facilitation of discrimination with forced trials in the present study resulted, at least in part, from the frequent alternation involved. Consideration of such a factor does not, of course, discount the possible contribution of added experience with the LFA for this group. Clearly, additional research manipulating these two variables independently will be required to assess their relative contribution to the enhancement of discrimination performance.

\section{REFERENCES}

CLAYTON, $K$, N. T-maze acquisition and reversal as a function of intertrial interval. Journal of Comparative \& Physiological Psychology, 1966, 62, 409-414.

LACHMAN, $R$. The influence of thirst and schedules of reinforcementnonreinforcement ratios upon brightness discrimination. Journal of Experimental Psychology, 1961, 62, 80-87.

LESTER, D. Spontaneous alternation and learning in rats. Psychonomic Science, 1967, 9, 575-576.

\section{NOTES}

1. This research was supported by Predoctoral Fellowship 2-F1-MH-30, 415-04 (PS) and Research Grant MH12064 from the National Institute of Mental Health.

2. Now at Franklin and Marshall College.
DOTY, B. A. Age differences in avoidance conditioning as a function of distribution of trials and task difficulty. The Journal of Genetic Psychology, 1966, 109, 249-254.

DOUGLAS, R. J., \& KOWAL, D. Spontaneous alternation and the brain. Paper presented at 48th meeting of Western Psychological Association

KASPER, P. Attenuation of passive avoidance by continuous septal stimulation. Psychonomic Science, 1964, 1, 219-220.

KIMBLE, D. P., KIRKBY, P. J., \& STEIN, D. G. Response perseveration interpretation of PA deficits in hippocampal rats. Journal of Comparative \& Physiological Psychology, 1967, 61, 141-143.

KIRBY, R. H. Acquisition, extinction and retention of an avoidance response in rats as a function of age. Journal of Comparative \& Physiological Psychology, 1963, 56, 158-162.

SCHWARTZBAUM, J. S., \& SPIETH, T. M. Analysis of the response-inhibition concept of septal functions in PA behavior. Psychonomic Science, 1964, 1, 145-146.

SIEGEL, S. Nonparametric statistics for the behavioral sciences. New York: McGraw-Hill, 1956.

NOTE

1. The author would like to thank Dr. William N. Dember for his critical reading of this paper. 